\title{
lonic Liquids in Charge Storage Devices: Effect of Purification on Performance
}

\author{
Stenger-Smith, John D., Chafin, Andrew P., Kline Jr., Clare F., \\ Ostrom, Gregory S. and Quintana, Roxanne L. \\ NAWCWD Chemistry Division, China Lake CA
}

USA

\section{Introduction}

Ionic liquids have been the subject of study over the past several years and there are quite a few advantages to their use in charge storage devices such as extremely low volatility, high voltage window, and inherently high concentration of cations and anions for charge transport processes ${ }^{1-8}$. Several ionic liquids are also commercially available. Purity of ionic liquids has been the subject of several publications-11. The impurities (chloride or water for example) discussed in these publications have a profound effect on the physical properties of the ionic liquids. There is a continuing discussion about the effect of ionic liquid purification on the electrochemical performance of electroactive charge storage devices.

In this chapter we examine the effect of column chromatography purification of ethyl methyl imidazolium bis(trifluoromethanesulfonylimide), (EMIBTI) (synthesized by our laboratory on the 100 gram scale) on the performance of a Type I electroactive polymer supercapacitor based upon poly(propylene dioxythiophene) (PProDOT).

The EMIBTI was purified on a 100-gram scale preparatory column using $20 \%$ acetonitrile and $80 \%$ chloroform as the eluting solvent.

A detailed analysis was performed on the ionic liquid and impurities such as water, lithium chloride, imidazolium, and other elements and compounds. Best efforts were made to quantify the amounts of these impurities. An attempt was made to correlate the presence or absence of an impurity with electrochemical growth and performance of the Type I electroactive supercapacitor.

\section{Experimental}

All electrochemical techniques were used as described previously12,13 and all experiments were performed in a dry box. ProDOT and EMIBTI were synthesized according to published procedures ${ }^{12}$. Chromatography (as described in the Introduction) was performed on the 50 gram scale and approximately $100 \mathrm{~mL}$ fractions were collected until about $90 \%$ of the original material was recovered. There was a dark band at the origin that remained at the top of column.

\subsection{Evaluation of capacitor performance}

The 2-electrode devices were assembled according to previously published reports ${ }^{12}$. The devices were then switched from 0 to 1.0 Volts at $0.1,1.0$, and 10.0 Volts per second to 
determine the initial charge capacity and voltage performance. The devices were then cycled for 10,000 cycles at 1.0 Volt per second (approximately 70\% depth of discharge). The devices were re-analyzed and the charge capacity and voltage performance was compared to initial values.

Another set of devices was prepared and the devices cycled to extreme over-voltage (2.5 Volts) for several hundred cycles to determine any differences.

\subsection{Electron microscopy of electrodeposited films}

Electron microscopy of the electrode ends employed a Zeiss EVO-50 SEM. Most images were taken using 6 kilo-volt $(\mathrm{kv})$ accelerating voltage and a calculated beam current of 10 to 25 picoamperes $(\mathrm{pA})$. Images were collected using either a Secondary Electron (SE) or Quadra-Pole Backscatter Detector (QBSD). The SE detector is the normal imaging detector (it shows surface topography) and the QBSD shows gray scale differences based on average atomic weight (the higher the atomic weight the brighter the image area). Semi-quantitative chemical analysis was done by Energy Dispersive Spectroscopy (EDS) using an EDAX Model Leo-EVO-50EP 132-10 Spectrometer. Working distance was approximately $9 \mathrm{~mm}$. Locations on the electrode ends before coating and after coating were imaged. After coating, areas of thin coating as well as areas of thick coating were compared and contrasted.

\subsection{Metals analysis of ionic liquids}

Approximately 1 gram of each ionic liquid was weighed out to within $0.0001 \mathrm{~g}$ into separate $50-\mathrm{mL}$, polypropylene digestion vials. Approximately $15 \mathrm{~mL}$ of concentrated nitric acid (trace metals grade) was added to the vials. The vials were covered with a polypropylene watch glass and allowed to reflux at $\sim 90^{\circ} \mathrm{C}$ for 2 hours. The samples were cooled and brought to a final volume of $100 \mathrm{~mL}$ in polypropylene volumetric flasks. The solutions were then analyzed for metals.

The samples were analyzed on a TJA IRIS Advantage inductively coupled plasma atomic emission spectrometer (ICP-AES) interfaced to a COMPAQ Deskpro computer using ThermoSPEC software for data collection. The system was calibrated from 0 to $10 \mathrm{ppm}$ using a certified calibration standard. The calibration curve was verified using a certified 1 ppm check standard. Each sample was aspirated into an argon plasma using an autosampler, a peristaltic pump, and a nebulizer. All elements were analyzed simultaneously. For each element, three replicate emission intensities were measured by the charge injection detector in the spectrometer, averaged, and converted to solution concentrations using the established calibration curve. A detection limit of $100 \mathrm{ppb}$ (ug/L) was established for the analysis.

\subsection{Anion analysis of ionic liquids}

Approximately 1 gram of each ionic liquid was weighed out to within $0.0001 \mathrm{~g}$ into separate $50-\mathrm{mL}$ polyethylene centrifuge tubes. Exactly $20 \mathrm{~mL}$ of 18 Megaohm deionized water was pipetted into each the vial. The vials were capped, shaken vigorously for 1 minute, and then sonicated for 2 hours. The vials were then centrifuged for 1 hour at $5000 \mathrm{rpm}$. The supernatant liquids were analyzed for anions by ion chromatography.

A Dionex ICS-2500 High Performance Ion Chromatograph with Eluent Generator was used to perform the anion analyses. This was accomplished using a Dionex AS4A analytical column coupled to a Dionex AG4A guard column. A 2-mL/min flow rate of $30 \mathrm{mM}$ 
hydroxide solution was produced electrolytically by the eluent generator using a $\mathrm{KOH}$ cartridge. Samples were injected onto the column using a 25 microliter sample loop. The background was suppressed electrolytically using an anionic self-regenerating suppressor $(193 \mathrm{~mA})$ and $18 \mathrm{Megaohm}$ deionized water at a flow rate of $4 \mathrm{~mL} / \mathrm{min}$. Analyte peaks were detected using a conductivity detector. The chromatograph was interfaced to a Dell GX260 Pentium 4 computer using Windows XP and Chromeleon software (Version 6.50) for data acquisition and manipulation. Calibration curves for each analyte were generated using linear least squares best fit lines generated from the peak area of the standards.

\subsection{Extraction of ionic liquids and analysis of purification column residue}

The non-chromatographed and chromatographed EMIBTI were extracted with hexane. Methylene chloride, as well as other solvents were attempted, but all were miscible with the ionic liquid. Approximately $1 \mathrm{~mL}$ of each sample was added to approximately $1 \mathrm{~mL}$ of HPLC grade hexanes. The samples were thoroughly shaken and allowed to separate overnight (hexanes on top, ionic liquids on the bottom). The upper layer of hexanes was removed, and analyzed using GC/MS. Approximately $1 \mu \mathrm{L}$ of the sample was injected into a gas chromatograph (Agilent 6890 with a Restek RTX-5MS 30-meter column) with a mass selective detector (Agilent 5973) for analysis. GC oven conditions were 3 minutes at $40^{\circ} \mathrm{C}$ and then increased to $350^{\circ} \mathrm{C}$ at $10^{\circ} \mathrm{C} /$ minute.

In another experiment, the resultant brown residue in the chromatography column (the top layer) was extracted with methanol to give about 0.01 grams of a tan solid. This material was analyzed using Mass Spectrometry and Nuclear Magnetic Resonance.

\section{Results}

The effect of the purity of the ionic liquid on the electrochemical growth of the PProDOT layers was compared using standard electrochemical techniques. Figure 1 shows the growth characteristics of PProDOT grown in the non-chromatographed ionic liquid. There were no discernable differences between growth characteristics of films grown using the nonchromatographed and the chromatographed ionic liquid.

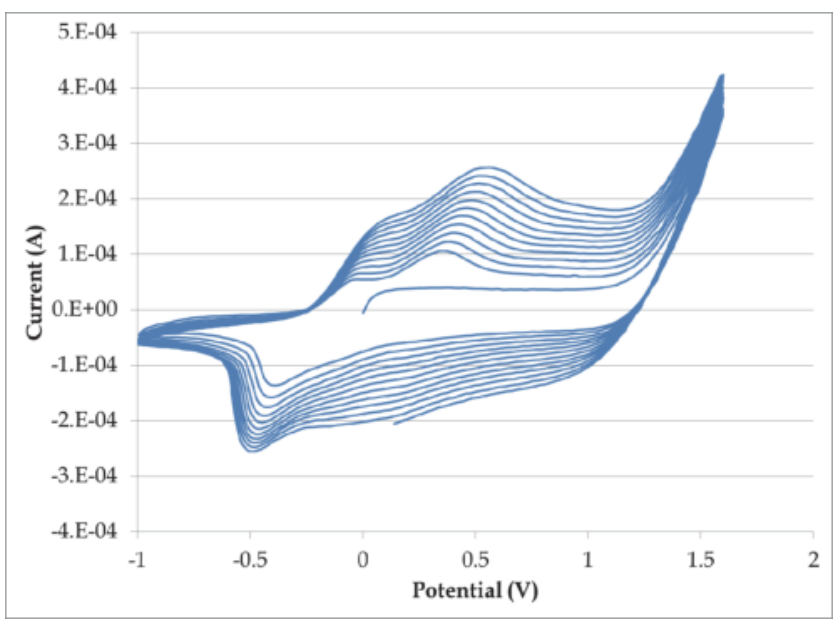

Fig. 1. Cyclic Voltammogram of PProDOT performed in unpurifed EMIBTI. 


\subsection{Capacitor performance}

The results shown in Figure 2 indicate there appears to be very little, if any difference between the initial electrochemical behaviour of the devices that were grown and cycled in chromatographed EMIBTI and devices that were grown and cycled in nonchromatographed EMIBTI.

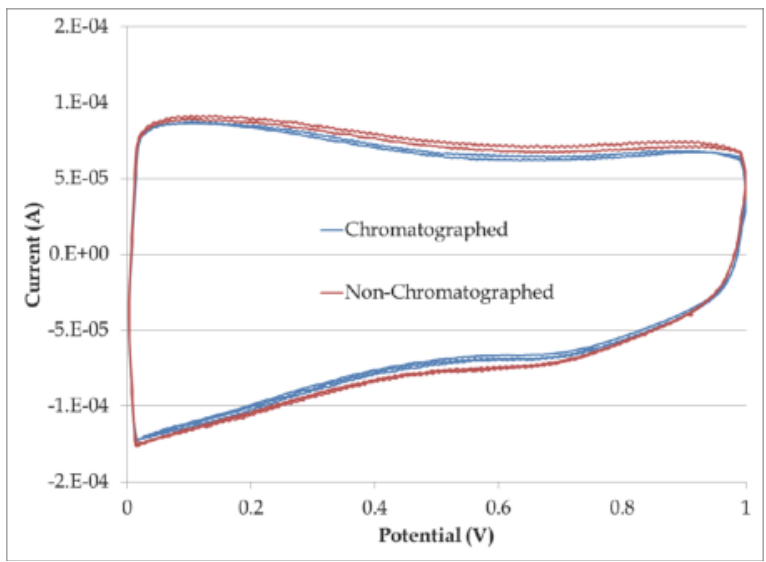

Fig. 2. Electrochemical behaviour comparison of supercapacitors using chromatographed (blue) and non-chromatographed (red) EMIBTI.

The long-term cycling performance of supercapacitors using the different grades of EMIBTI was then examined. As seen in Figure 3, devices grown and cycled using chromatographed EMIBTI improved long-term behaviour as far as charge retention and the ability to operate (store and release charge) at the maximum potential of 1 Volt.

Figure 3 shows that the electrochemical behaviour of the supercapacitor using chromatographed EMIBTI after 10,000 full cycles is nearly identical to that of the original (in Figure 2); whereas the electrochemical behaviour of the supercapacitor using the nonchromatographed sample is significantly degraded after 10,000 full cycles.

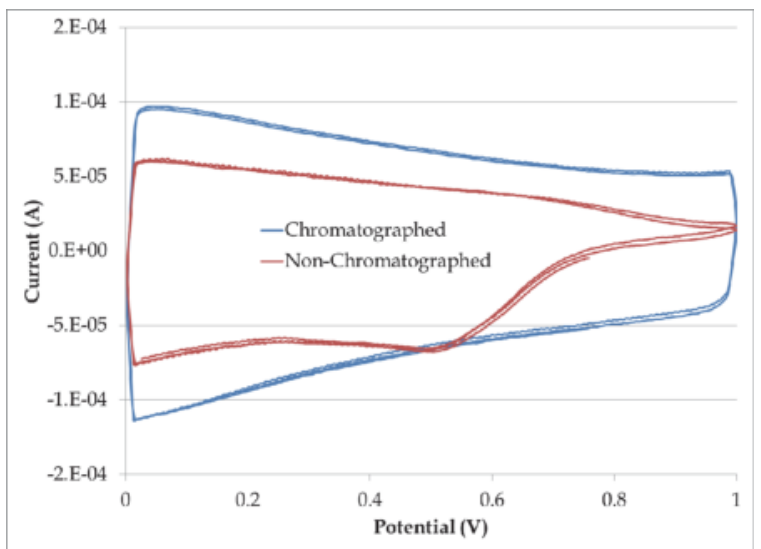

Fig. 3. Electrochemical behaviour comparison of supercapacitors after 10,000 cycles using chromatographed (blue) and non-chromatographed (red) EMIBTI. 
In order to elucidate the effect of the growth versus the cycling electrolyte, the same sets of experiments and analysis were performed on capacitors in which the PProDOT was grown in the chromatographed and cycled in the non-chromatographed, and on capacitors in which the PProDOT was grown in the non-chromatographed and cycled in the chromatographed. Table 1 summarizes the results of all 4 possibilities.

\begin{tabular}{|c|c|c|c|c|}
\hline $\begin{array}{c}\text { Growth } \\
\text { Medium }\end{array}$ & $\begin{array}{c}\text { Cycle } \\
\text { Medium }\end{array}$ & $\begin{array}{c}\text { Initial Max } \\
\text { Potential } \\
\text { (V) }\end{array}$ & $\begin{array}{c}\text { Potential } \\
\text { After 10 K } \\
\text { (V) }\end{array}$ & $\begin{array}{c}\text { Normalized } \\
\text { Capacity } \\
\text { (10K) }\end{array}$ \\
\hline Chromatographed & Chromatographed & 1.0 & 1.0 & 0.93 \\
\hline $\begin{array}{c}\text { Non- } \\
\text { chromatographed }\end{array}$ & $\begin{array}{c}\text { Non- } \\
\text { chromatographed }\end{array}$ & 1.0 & 0.7 & 0.53 \\
\hline Chromatographed & $\begin{array}{c}\text { Non- } \\
\text { chromatographed }\end{array}$ & 0.7 & 0.65 & $0.88^{*}$ \\
\hline $\begin{array}{c}\text { Non- } \\
\text { chromatographed }\end{array}$ & Chromatographed & 1.0 & 1.0 & 0.64 \\
\hline
\end{tabular}

*Initial capacity was 30\% lower

Table 1. Summary of dependence of performance on growth and switching electrolyte.

Interestingly, devices that were grown in the chromatographed EMIBTI and cycled in the non-chromatographed EMIBTI showed an immediate lack of performance at the full device potential, with $30 \%$ lower on average initial capacity, which is shown in Figure 4 . The devices that were both grown and cycled in the non-chromatographed EMIBTI didn't lose the full cycle potential until after more than 2000 cycles.

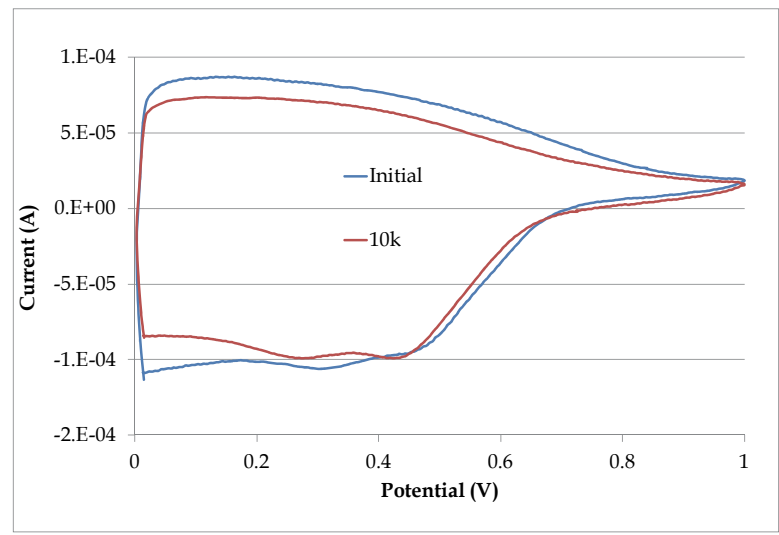

Fig. 4. Initial- (blue) and long-term (10k cycles red) electrochemical behaviour of supercapacitors using chromatographed EMIBTI as the growth electrolyte and nonchromatographed EMIBTI as the cycling electrolyte.

There was a slight decrease in the overall voltage, along with a $12 \%$ loss in initial capacity after 10,000 cycles. If the initial capacity loss is included, it can be qualitatively concluded that the overall capacity loss is about the same as those films cycled and grown in both the non-chromatographed EMIBTI. 
Devices that were grown in the non-chromatographed EMIBTI then cycled in the chromatographed EMIBTI showed a 36\% loss in capacity after 10,000 cycles but still retained the ability to move and store charge at 1.0 Volts.

The over-voltage behaviour of the devices using each grade of EMIBTI was compared. Devices were cycled to well past the potential where PProDOT stores and releases energy. Each system was subject to over-voltage at 2.5, 3.0 and 3.5 volts for 100 cycles at $90 \%$ depth of discharge. All samples, regardless of whether the EMIBTI was chromatographed, decayed rapidly, and there appeared to be no quantifiable difference between samples.

\subsection{Electron microscopy of electrodeposited films}

The properties of the films from each grade of EMIBTI were elucidated using Scanning Electron Microscopy to determine whether there are any significant and quantifiable differences between the architecture of the films. Figures 5 and 6 are typical Electron Micrographs of the PProDOT polymer films deposited shown at 15 and 500 times magnification. There was no discernable difference in the film topography by electron microscopy between films grown with the chromatographed and films grown with the nonchromatographed EMIBTI. Note that the polymer film is deposited in the circular area in the 15X magnification. The rest of the photo shows the poly(tetrafluoroethylene) casing of the electrode. The image quality was adversely affected since the intact, $158 \mathrm{~mm}$ long and 61.6 gram electrode needed to be in the chamber (a rather unique circumstance for SEM) to properly image the electrode end coated with the PProDOT film.

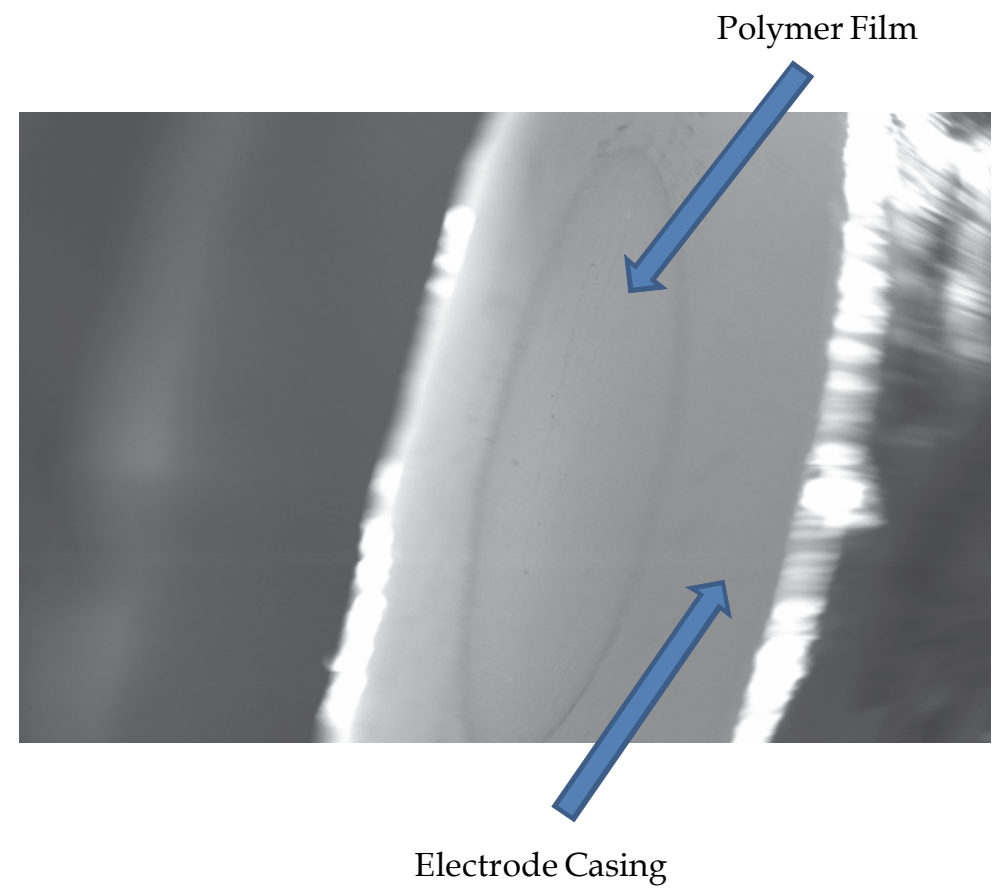

Fig. 5. Electron Micrograph of PProDOT film 15X (grown in non-chromatographed EMIBTI). 


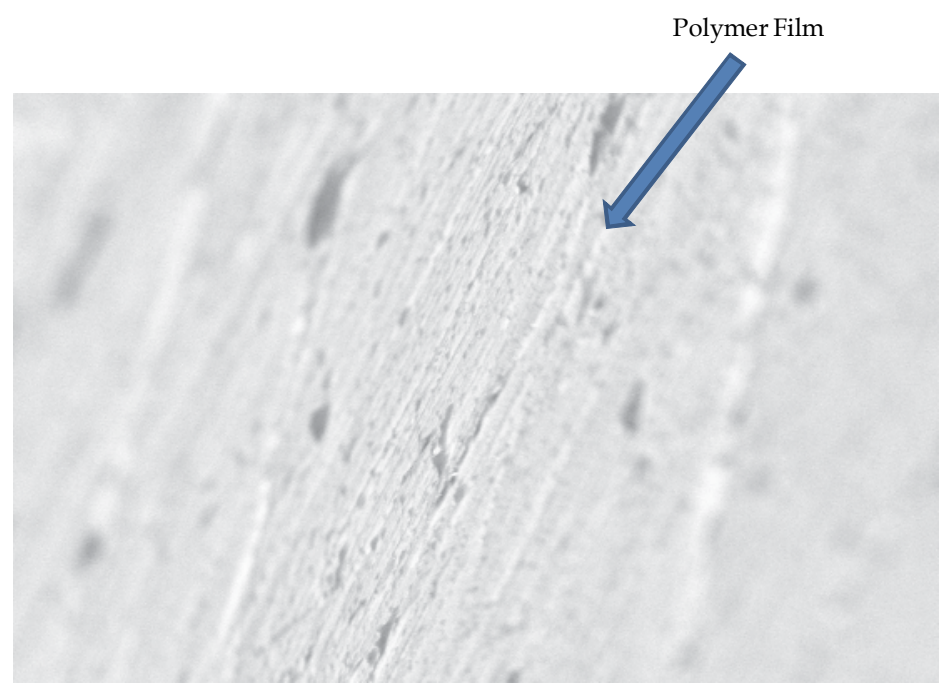

Fig. 6. Electron Micrograph of PProDOT film 500X (grown in non-chromatographed EMIBTI).

Analysis using EDS elemental analysis determined that there is no significant difference in the incorporation of key elements ${ }^{13}(\mathrm{C}, \mathrm{N}, \mathrm{O}, \mathrm{F}$ and $\mathrm{S}$ for example) into the polymer layer as well as any traces of impurities such as $\mathrm{Cl}$. At this semi-quantitative scale it could not be determined whether the amounts of impurities in the EMIBTI samples correlated with any impurities found in the polymer films. Figure 7 is an overlay of the EDS of polymer films grown in chromatographed and non-chromatographed EMIBTI, and shows that the elemental profiles of each film are for all practical purposes identical. These results support the hypothesis that the impurities in the non-chromatographed EMIBTI degrade the longterm switching of devices and not necessarily the short-term behaviour and/or the electrochemical growth of PProDOT.

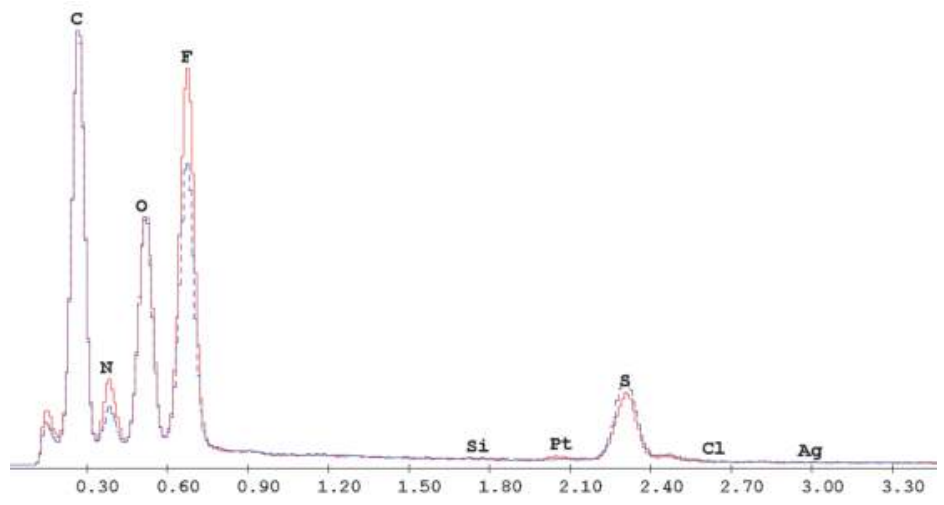

Fig. 7. Overlay of the EDS Spectra of polymer films chromatographed (blue) and nonchromatographed (red). 
Neither film had any measureable amount of impurity such as chloride. Platinum was often detected over the area of measurement, but this was due to the thinness of the film on the platinum substrate and the x-ray interaction volume caused by the electron beam.

\subsection{Metals analysis of ionic liquids}

The samples were analyzed using the previous protocol for the following elements: $\mathrm{Al}, \mathrm{As}$, B, Ba, Be, Ca, Cd, Co, Cr, Cu, Fe, K, Li, Mg, Mn, Mo, Na, Ni, Pb, Sb, Se, Si, Sn, Sr, Ti, Tl, V and $\mathrm{Zn}$. The detection limit for these elements is $100 \mathrm{ppb}$. Sodium, $\mathrm{Na}$, was the only element that was detected: $210 \mathrm{ppm}$ in the non-chromatographed sample and $290 \mathrm{ppm}$ in the chromatographed sample. The propylene carbonate, used in the electrochemical growth of the polymer films, was also analyzed and found to have no detectable impurities at this reporting limit. These results for the most part are not particularly surprising since both EMIBTI samples were extracted many times with water. The lack of Li present in either sample is quite significant, as $\mathrm{Li}^{+}$is a major component of one of the starting materials, and could have an effect on the electrochemical properties.

\subsection{Anion analysis of ionic liquids}

Chloride and fluoride were detected above the threshold in each sample. This technique detected $1.25 \mathrm{ppm}$ chloride and $2.23 \mathrm{ppm}$ fluoride in the non-chromatographed sample and $0.265 \mathrm{ppm}$ chloride and $0.401 \mathrm{ppm}$ fluoride in the chromatographed sample. This result is noteworthy in that chloride is well-established as an undesirable impurity in ionic liquids and can affect many factors 10,11 , not just electrochemistry.

\subsection{Extraction of ionic liquids and analysis of purification column residue}

The hexane extracts of both samples were clear and colorless. The extract of the chromatographed ionic liquid had a higher concentration of common components extracted (possibly contaminants from the glue in the vial caps) and visible in the gas chromatogram than the non-chromatographed sample. However, there were a few components present in the non-chromatographed sample that were not in the chromatographed sample. Since these compounds were most likely removed during the purification. The impurities (non-common components) detected by this technique appeared to be degradation products of imidazole. The Mass Spectrometry analysis of the column residue was inconclusive. Only siloxanes (glassware grease and silica gel dissolved by the methanol) were detected, and only at very small levels.

Nuclear Magnetic Resonance Spectroscopy ( ${ }^{1} \mathrm{H}$ NMR) in $\mathrm{d}_{6}$ acetone indicated the presence of an ethyl methyl imidazolium salt. This was verified by the 2 single peaks at 7.80 and $7.79 \mathrm{ppm}$, a single signature peak at $9.41 \mathrm{ppm}$, a single methyl peak at $4.0 \mathrm{ppm}$ as well as the quartet-triplet signature peak of an ethyl group at 4.4 and $1.6 \mathrm{ppm}$ respectively. Comparison of this spectrum to ethyl methyl imidazolium chloride, with a signature peak at $10.84 \mathrm{ppm}$ and EMIBTI, with a signature peak at 9.02 leads to the conclusion that this imidazolium impurity is neither a pure chloride nor pure BTI salt of imidazolium, though it could be a mixture of chloride and BTI salts, or a completely different imidazolium compound.

NMR also indicated the presence of some type of hydrocarbon-based impurity which was difficult to identify. 


\section{Summary and conclusions}

The purity of ionic liquids was found to be a critical factor in the performance of electroactive polymer-based energy storage devices. The chemical identity of the electrodeposited films is for all practical purposes identical as determined by semiquantitative quantum backscatter experiments. Films grown and switched in the chromatographed ionic liquid retained the most activity at higher voltages and retained $93 \%$ of initial capacity after 10,000 cycles at 70\% depth of discharge. Films grown and switched in the non-chromatographed ionic liquid retained the least activity at higher voltages and retained only $53 \%$ of initial capacity after 10,000 cycles at $70 \%$ depth of discharge. Films grown in the non-chromatographed ionic liquid and switched in the chromatographed ionic liquid retain the ability to store and transfer charge at the upper end of the voltage window, but retained only $64 \%$ of the initial capacity. Films grown in the chromatographed ionic liquid and switched in the non-chromatographed ionic liquid degrade more slowly as those grown and switched in the non-chromatographed liquid (88\% capacity retained) but devices made from these films do not have the higher voltage (greater than 0.5 Volts), activity and the initial overall capacity of the films was consistently $30 \%$ lower.

Therefore, there must be some trace impurity in the non-chromatographed ionic liquid that is responsible for the performance degradation. This impurity affects the behaviour of the devices when it is used as a supporting electrolyte for the electrochemical growth, as well as an electrolyte for the device. Based upon the results using the chromatographed EMIBTI as the growth solvent and non-chromatographed EMIBTI as the cycling solvent, the incorporation of this impurity into the polymer film appears to significantly degrade the full device potential. Residual lithium ion can be ruled out for all practical purposes as it was well below $100 \mathrm{ppb}$ for both samples. Devices using both types of EMIBTI degraded very rapidly at severe overvoltage, due to the irreversible oxidation of PProDOT being nearly independent of electrolyte impurity.

The impurities possibly responsible for performance issues have been narrowed down to the following: 1) The presence of $40 \%$ more sodium in the chromatographed sample may be beneficial to device performance. As unlikely as this is, this cannot be ruled out at this time. 2) The presence of 5.5-fold more fluoride and 4.7-fold more chloride in the nonchromatographed sample. This is a likely source of the performance degradation given the effect of chloride and fluoride on electrochemical performance ${ }^{9}$. 3) The presence of an organic impurity, namely an imidazolium salt of an anion that is neither chloride nor bis(trifluoromethyl sufonylimide) salt. This imidazolium impurity was detected from the material left behind on the chromatography column and must also be considered a very likely source of performance degradation. 4) An impurity that was undetected and/or unidentifiable by the analytical techniques that were used in this study.

The removal of these impurities using the chromatography described in this paper and/or other purification techniques ${ }^{14}$ is recommended for electrochemical synthesis and analysis.

\section{References}

[1] Pringle, J. M., Forsyth, M., MacFarlane, D. R., Wagner, K., Hall, S. B., and Officer, D. L., (2005) Polymer, 46, 2047.

[2] Deepa, M., Awadhia, A., Bhandari, S., (2009) Chem. Phys. 11(27), 5674-5685.

[3] Ferrari, S.; Quartarone, E., Mustarelli, P., Magistris, A., Protti, S., Lazzaroni, S., Fagnoni, M., Albini, A. (2009) J. Power Sources, 194(1), 45-50 
[4] Zakeeruddin, S. M., . Graetzel, M., Adv. Funct. Mater. 2009, 19(14), 2187-2202.

[5] Armand, M., Endres, F., MacFarlane, D. R., Ohno, H., and Scrosati, B. (2009) Nature Mater., 8(8), 621-629.

[6] Fernicola, A., Scrosati, B., and Ohno, H. (2006) Ionics 12, 95-102.

[7] Ohno, H, (ed.), (2005) Electrochemical Aspects of Ionic Liquids, Wiley Interscience, Hoboken, NJ.

[8] Lu, W. Fadeev, A. G., Qi, B., Smela, E., Mattes, B. R., Ding, J., Spinks, G. M., Mazurkiewicz, J., Zhou, D., Wallace, G. G., MacFarlane, D. R., Forsyth, S. A., and Forsyth, M., (2002) Science 297, 983-987.

[9] Irvin, J. A. and Stenger-Smith, J. D., (2009) Aldrich Material Matters ${ }^{T M}$ Vol. 4, issue 4 on Alternative Energy

[10] Seddon, K. R., Stark, A., and Torres, M. J., (2000) J. Pure Appl. Chem. 72, 2275

[11] Zhang J., and Bond, A. M., (2005) The Analyst, 130, 1132

[12] Stenger-Smith, J., Webber C. K., Anderson N., Chafin, A.P., Zong, K and. Reynolds, J. R., (2002) J. Electrochem. Soc. 149(8), A973-A977

[13] Stenger-Smith, J. D., Guenthner, A., Cash, J., Irvin, J. A., and Irvin, D. J., (2010) J. Electrochem. Soc. 157 (3) A298

[14] Rosario Canales, M. R., Pravas, D., Therien, M. J., Gopu, P., Sotero-Esteva, J. O., Santiago-Aviles J. J., (2008) Single-Wall Carbon Nanotube/Poly(p-napththalene ethynylene) Composites as Electtrode Materials for Supercapacitor Applications http:/ / xilonen.inaoep.mx/revista_electronica/volumen/descarga_articulo.php?ar ticulo $=c 1-04-I B 08 . p d f \& i d a=$ cedebb6e872f539bef8c3f919874e9d7 


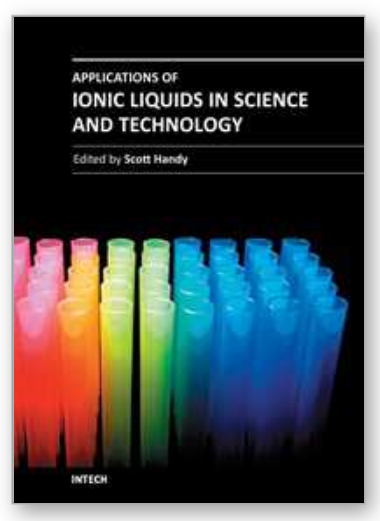

\author{
Applications of lonic Liquids in Science and Technology \\ Edited by Prof. Scott Handy
}

ISBN 978-953-307-605-8

Hard cover, 516 pages

Publisher InTech

Published online 22, September, 2011

Published in print edition September, 2011

This volume, of a two volume set on ionic liquids, focuses on the applications of ionic liquids in a growing range of areas. Throughout the 1990s, it seemed that most of the attention in the area of ionic liquids applications was directed toward their use as solvents for organic and transition-metal-catalyzed reactions. Certainly, this interest continues on to the present date, but the most innovative uses of ionic liquids span a much more diverse field than just synthesis. Some of the main topics of coverage include the application of RTILs in various electronic applications (batteries, capacitors, and light-emitting materials), polymers (synthesis and functionalization), nanomaterials (synthesis and stabilization), and separations. More unusual applications can be noted in the fields of biomass utilization, spectroscopy, optics, lubricants, fuels, and refrigerants. It is hoped that the diversity of this volume will serve as an inspiration for even further advances in the use of RTILs.

\title{
How to reference
}

In order to correctly reference this scholarly work, feel free to copy and paste the following:

John D. Stenger-Smith, Andrew P. Chafin, Clare F. Kline Jr., Gregory S. Ostrom and Roxanne L. Quintana (2011). Ionic Liquids in Charge Storage Devices: Effect of Purification on Performance, Applications of lonic Liquids in Science and Technology, Prof. Scott Handy (Ed.), ISBN: 978-953-307-605-8, InTech, Available from: http://www.intechopen.com/books/applications-of-ionic-liquids-in-science-and-technology/ionic-liquids-incharge-storage-devices-effect-of-purification-on-performance

\section{INTECH}

open science | open minds

\author{
InTech Europe \\ University Campus STeP Ri \\ Slavka Krautzeka 83/A \\ 51000 Rijeka, Croatia \\ Phone: +385 (51) 770447 \\ Fax: +385 (51) 686166 \\ www.intechopen.com
}

\author{
InTech China \\ Unit 405, Office Block, Hotel Equatorial Shanghai \\ No.65, Yan An Road (West), Shanghai, 200040, China \\ 中国上海市延安西路65号上海国际贵都大饭店办公楼 405 单元 \\ Phone: +86-21-62489820 \\ Fax: +86-21-62489821
}


(C) 2011 The Author(s). Licensee IntechOpen. This chapter is distributed under the terms of the Creative Commons Attribution-NonCommercialShareAlike-3.0 License, which permits use, distribution and reproduction for non-commercial purposes, provided the original is properly cited and derivative works building on this content are distributed under the same license. 